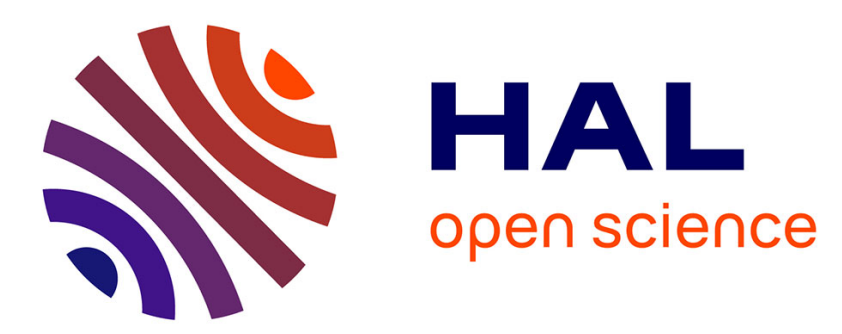

\title{
Attractor Equivalence: An Observational Semantics for Reaction Networks
}

\author{
Guillaume Madelaine, Cédric Lhoussaine, Joachim Niehren
}

\section{To cite this version:}

Guillaume Madelaine, Cédric Lhoussaine, Joachim Niehren. Attractor Equivalence: An Observational Semantics for Reaction Networks. First International Conference on Formal Methods in MacroBiology, Sep 2014, Nouméa, New Caledonia. pp.82-101. hal-00990924

\section{HAL Id: hal-00990924 https://hal.science/hal-00990924}

Submitted on 9 Jul 2015

HAL is a multi-disciplinary open access archive for the deposit and dissemination of scientific research documents, whether they are published or not. The documents may come from teaching and research institutions in France or abroad, or from public or private research centers.
L'archive ouverte pluridisciplinaire HAL, est destinée au dépôt et à la diffusion de documents scientifiques de niveau recherche, publiés ou non, émanant des établissements d'enseignement et de recherche français ou étrangers, des laboratoires publics ou privés. 


\title{
Attractor Equivalence: An Observational Semantics for Reaction Networks
}

\author{
Guillaume Madelaine $^{1,2}$, Cédric Lhoussaine ${ }^{1,2}$, Joachim Niehren $^{1,3}$ \\ 1 BioComputing, LIFL Lille (CNRS UmR 8022) \\ 2 University of Lille 1, France \\ 3 InRIA Lille, France
}

\begin{abstract}
We study observational semantics for networks of chemical reactions as used in systems biology. Reaction networks without kinetic information, as we consider, can be identified with Petri nets. We present a new observational semantics for reaction networks that we call the attractor equivalence. The main idea of the attractor equivalence is to observe reachable attractors and reachability of an attractor divergence in all possible contexts. The attractor equivalence can support powerful simplifications for reaction networks as we illustrate at the example of the Tet-On system. Alternative semantics based on bisimulations or traces, in contrast, do not support all needed simplifications.
\end{abstract}

\section{Introduction}

A reaction network is pair that consists of a system of chemical reactions and a initial chemical solution, to which the reactions are to be applied. The kinetics of a chemical reaction defines its speed, in function of the chemical solution to which it is applied. Reaction networks can be considered as programs of a programming language, whose operational semantics describes the evolution of the initial chemical solution over time. A prominent programming language for chemical reaction networks is BioCham [4], but there are also programming language for more powerful biochemical reaction networks such as Kappa [5] and React(C) [22].

Reaction networks have three kinds of operational semantics (see e.g. [9,32]). The stochastic semantics of a reaction network is a continuous time Markov chain which for any chemical solution $S$ and any time point $t$ defines the probability of reaching $S$ at $t$. Stochastic simulation algorithms generate the traces of a reaction network according to its stochastic semantics. The deterministic semantics of a reaction network is a system of ordinary differential equations, that tries to approximate the average concentration of the chemical solutions at any time point. It is the input of deterministic simulation algorithms. The third qualitative semantics is independent of any kinetics. It is given by a binary relation, which states how a chemical solution can be reduced in a nondeterministic manner to another chemical solution by applying a reaction, and thus which solutions can be reached from the initial solution. 
In the following, we will stick to the qualitative semantics of reaction networks, i.e., we will ignore all kinetic informations. This is a simplification, which broadens the scope of our approach (since the kinetics are often unknown) but at the cost of lower precision. As a consequence, reaction networks can be identified with Petri nets. The same simplification was adapted in much previous work on static analysis of reaction networks (see e.g. $[45,36,42]$ ), either since the kinetics doesn't matter for the question under consideration, or also, since the precise kinetics are unknown.

The observational semantics of a program is usually defined on top of operational semantics, with the objective to formalize its input-output behaviour $[31,37,39]$. This is done by defining a notion of program equivalence, so that equivalent programs can be exchanged by each other in any admissible context without affecting the observable behavior. The observations of the output produced by equivalent reaction networks must thus be the same in all admissible contexts (which generalize on inputs). However, there exists no notion of observational semantics for reaction networks so far. The missing ingredients are appropriate notions of observations and admissible contexts. In particular, one cannot rely on observing termination, as in all previous work on observational semantics of functional programming languages, since biological systems may change without end during an oscillation or in an equilibrium.

In this paper we propose an observational equivalence for reaction networks, that we call the attractor equivalence. The main idea is to observe reachable attractors, i.e., strongly connected components of chemical solutions so that each of them can be reached from each other. Note that whenever an attractor is reached, then the reduction must continue to loop infinitely within the attractor or terminate if the attractor is a singleton. Our semantics may also observe the reachability of an attractor divergence, i.e., of a chemical solution from which no attractor can be reached. A biological example of an attractor divergence is a tumor that is growing in an irreversible manner without termination. Furthermore, the observation of a chemical solution may not be able to see all informations about all molecules. What can be observed is fixed by a parameter that we call the observation function. In a biological example, one may be able to observe only fluorescent proteins under a microscope, or be unable to distinguish two different kinds of fluorescent proteins. A context of a reaction network is itself a reaction network. Rather than admitting all contexts, we fix as a parameter a subset of "admissible" contexts, by restricting the set of molecules that these contexts may affect. This corresponds for instance to biological experiments, in which only particular molecules in the environment of a cell can be added or consumed by a microfluidic device [46], but not any other molecule, in particular not those which are produced of consumed exclusively inside the cell. Finally, for a fixed observation function and a fixed set of admissible contexts, we call reaction networks attractor equivalent, if they have the same observations in all admissible contexts.

We also provide a set of axioms that we prove to be correct for the attractor equivalence, and justify their relevance at the example of a biological system. 
We have chosen a simplistic reaction network for the Tet-On system $[20,15]$ with only 10 reactions. It models the addition of Dox-molecules to the environment of a cell (by a microfluidic device), its transport into the cell, and the expression of the fluorescent molecule $G F P_{a}$, that can be observed by a microscope. We show that we can use our axioms to reduce the Tet-On network into an attractor equivalent network with only 2 reactions:

$$
D o x \rightarrow D o x+G F P_{a} \quad \text { and } \quad G F P_{a} \rightarrow \emptyset
$$

The only molecule that the context is admitted to affect is Dox. In any context $n$ Dox where $n \geq 1$, this reaction network system reaches a single attractor $\left\{n D o x+m G F P_{a} \mid m \geq 0\right\}$. Note that this wouldn't be true without the degradation reaction. The number $m$ of $G F P_{a}$ molecules can be observed in order to measure the degree of fluorescence, which may vary from 0 to infinity in the attractor. In the empty context, the above reaction network terminates immediately with the empty solution, so the singleton attractor $\left\{0 D o x+0 G F P_{a}\right\}$ is reached, of which the absence of $G F P_{a}$ is observed.

Even though very simple, the application to the Tet-On system already illustrates the appropriateness of the attractor equivalence, in that it is sufficiently powerful to support the needed simplifications. This is in contrast to alternative notions of program equivalences for Petri net which are either based on bisimulations or traces $[25,30,18]$. It turns out that 2 of our 5 axiom used for simplification are incorrect for these alternative equivalences. The problem is that these axioms change the internal cascades from the input to the output, which spoils bisimulation and alters traces. Conversely, neither the bisimilation equivalence nor the trace equivalence are included in the attractor equivalence, since attractor divergent networks may be equivalent there to non attractor divergent networks. Therefore, these alternative equivalences are indeed inappropriate.

Outline. We recall reaction networks with the qualitative semantics in Section 2 and define the attractor equivalence in Sections 3 and 4. In Section 5, we present a set of axioms of the attractor equivalence. In Section 6 we use them to simplify the detailed Tet-On system from [20]. Alternative equivalence notions for reaction networks are presented in Section 7 and more related work is discussed in Section 8. We then conclude with some future work in Section 9.

\section{Reaction networks}

We introduce of reaction networks without kinetic functions and define their qualitative operational semantics.

Let $\mathbb{N}_{0}$ be the set of natural numbers including 0 . We fix a set Spec of molecular species that will be ranged over by $A, B, C$.

A (chemical) solution $s \in$ Sol $:$ Spec $\rightarrow \mathbb{N}_{0}$ is a multiset of molecules. Give natural numbers $n_{1}, \ldots, n_{i}$, we denote by $n_{1} A_{1}+\ldots+n_{k} A_{k}$ the solution that contains $n_{i}$ molecules of species $A_{i}$ for all $1 \leq i \leq n$ and 0 molecules of all other 
species. We will write $s \backslash A$ for the solution obtained from solution $s$ by removing all molecules of species $A$, i.e., $s \backslash A(A)=0$ and $s \backslash A(B)=s(B)$ otherwise.

A (chemical) reaction $r \in S o l \times S o l$ is a pair of two solutions. We write $s_{1} \rightarrow s_{2}$ for the reaction $\left(s_{1}, s_{2}\right)$, and say that $s_{1}$ is the solution of reactants and $s_{2}$ the solution of products. We denote by $p r_{r}(A)=s_{2}(A)-s_{1}(A)$ the number of molecules $A$ produced by $r$. If this number is negative, than the molecules are consumed. In order to emphasize the enzymatic part of a reaction, we will write $s_{1} \stackrel{s_{3}}{\longrightarrow} s_{2}$ as a shorthand for the reaction $s_{1}+s_{3} \rightarrow s_{2}+s_{3}$. It will sometimes be conveniant to have a syntax for reversible reactions. To this end, we will write $s_{1} \stackrel{s_{3}}{\longrightarrow} s_{2}$ instead of the set with the two reactions $s_{1} \stackrel{s_{3}}{\longrightarrow} s_{2}$ and $s_{2} \stackrel{s_{3}}{\longrightarrow} s_{1}$.

Definition 1. A reaction network is a pair $\left\langle s_{0}, R\right\rangle$ consisting of a solution $s_{0}$ that we call the initial solution, and a finite set of reactions $R$.

Example 1. We consider the simplified Tet-On simple reaction network. In this case, the set of molecular species is $S p e c=\left\{D o x, G F P_{a}\right\}$, and the network contains the following two reactions:

$$
D o x \rightarrow D o x+G F P_{a} \quad \text { and } \quad G_{F P} \rightarrow \emptyset
$$

The first reaction produces active green fluorescence protein $G F P_{a}$ if the enzyme doxycycline $(D o x)$ is present outside of the cell. It can also be written as $\emptyset \stackrel{D o x}{\longrightarrow} G F P_{a}$. In reality, this reaction is performed by a cascade of reactions which transport Dox into the cell in order to trigger the expression of $G F P_{a}$ by transcription and translation. The second reaction degrades $G F P_{a}$ by consuming one $G_{F P}{ }_{a}$ molecule at each application. Degradation is a necessary reaction for making the system working, since otherwise, it would produce unlimited amounts of $G_{F P}$ without reaching an equilibrium and thus eventually explode the cell.

The qualitative operational semantics of reactions is defined by a binary relation on solutions, that states how a single reaction can be applied in a nondeterministic manner. Given a set of reactions $R$, we define the reduction relation $\vec{R}$ as the least binary relation that satisfies the following inference rule:

$$
\frac{s_{1} \rightarrow s_{2} \in R \quad s \in S o l}{s_{1}+s \underset{R}{\rightarrow} s_{2}+s}
$$

Sometimes we freely write $s_{1} \underset{N}{\rightarrow} s_{2}$ instead of $s_{1} \underset{R}{\rightarrow} s_{2}$ if $N=\langle s, R\rangle$ for some $s$.

The set of solutions accessible from an initial solution $s$ by a set of reactions $R$ is $A c c_{R}(s)=\left\{s^{\prime} \mid s \vec{R}^{*} s^{\prime}\right\}$. We also sometimes write $A c c_{N}=A c c_{R}(s)$ if $N=\langle s, R\rangle$. For the simple reaction network Tet-On for instance, we have $\operatorname{Acc}_{\text {Tet-On }}(D o x)=\left\{\right.$ Dox $\left.+m G F P_{a} \mid m \in \mathbb{N}_{0}\right\}$.

Definition 2 (Attractor). Let $R$ be a set of reactions. We call a set of solution $S \subseteq S o l$ an attractor for $R$ if $\operatorname{Acc}_{R}(s)=S$ for all $s \in S$. We denote by $\mathcal{A}(R)$ the set of attractors for $R$. 
For instance, if its initial solution contains only a molecule of Dox, the Tet-On $n_{\text {simple }}$ network have the attractor $\left\{D o x+m G F P_{a} \mid m \in \mathbb{N}_{0}\right\}$ since $D o x+(m-1) G F P_{a} \rightarrow D o x+m G F P_{a}$ and conversely. The same set, however, is not an attractor of $\left\{D o x \rightarrow D o x+G F P_{a}\right\}$, since there, one can never come back from Dox $+m G F P_{a}$ to $D o x+(m-1) G F P_{a}$.

\section{Context-less attractor equivalence}

We define a notion of context-less attractor equivalence for reaction networks, that we will lift to a context-sensitive version in the next section.

We first define an observation function that states what can be observed from an output chemical solution. For instance, we may want to express the fact that some proteins cannot be distinguished or that some other molecules cannot be seen at all.

Definition 3. An observation function $\Omega$ is a homomorphism from the monoid $($ Sol,,$+ \emptyset)$ to some other monoid.

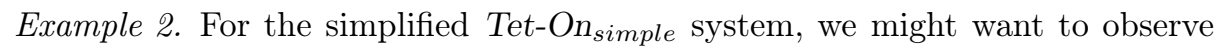
with a microscope the number of fluorescing molecules $G F P_{a}$. In this case, we map chemical solutions to natural numbers, i.e., to the monoid $\left(\mathbb{N}_{0},+, 0\right)$.

$$
\Omega_{\text {Tet-On }}\left(m G F P_{a}+s\right)=m \quad \text { where } G F P_{a} \notin s
$$

Alternatively, we may also want to observe the presence or absence of $G F P_{a}$. This can be done by using the Boolean monoid $(\{0,1\}$, max, 0$)$ as follows.

$$
\Omega_{\text {Tet-On }}^{\prime}\left(m G F P_{a}+s\right)=\max (m, 1) \quad \text { where } G F P_{a} \notin s
$$

Or else, we might want to count both $G F P_{a}$ and Dox molecules, since the latter can also be controlled experimentally (by the microfluedic device). In this case, we can use $(S o l,+, \emptyset)$ as source and target monoid as follows:

$$
\Omega_{\text {Tet-On }}^{\prime \prime}\left(m G F P_{a}+n D o x+s\right)=m G F P_{a}+n D o x \quad \text { where } G F P_{a}, \text { Dox } \notin s
$$

We next define our observation predicates which will be based on the accessibility of attractors. We fix an observation function $\Omega$ and let $O \subseteq \Omega(S o l)$ be a set of observations.

Definition 4. We say that a network $\left\langle s_{0}, R\right\rangle$ may converge to $O$ if it may reach an attractor with observables $O$ :

$$
\left\langle s_{0}, R\right\rangle \downarrow ⿱ \text { iff } \exists S \in \mathcal{A}(R) \text { s.t. } S \subseteq A c c_{R}\left(s_{0}\right) \text { and } \Omega(S)=O
$$

An attractor divergence is a solution that may not converge to any set of observations. We say that a network may diverge if it can reach an attractor divergence.

$$
\left\langle s_{0}, R\right\rangle \uparrow \text { iff } \exists s \in \operatorname{Acc}_{R}\left(s_{0}\right) \text { s.t. } \neg \exists O .\langle s, R\rangle \downarrow_{O}
$$




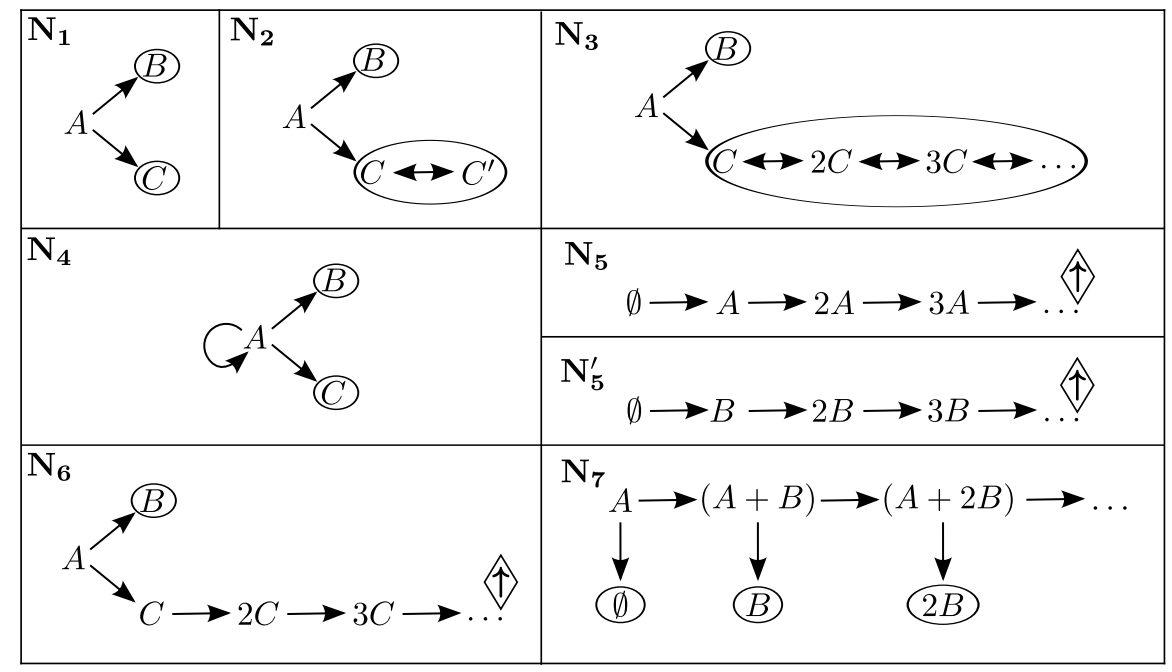

Fig. 1: Accessibility graphs of example reaction networks. The ovals denote attractors, and $\uparrow$ indicates a divergence. The networks $N_{1}, N_{2}, N_{3}$, and $N_{4}$ must converge to either $\{B\}$ or $\{C\}$ for the observation function from Example 3 which identifies $C^{\prime}$ with $C$ and ignores the multiplicities of $C$ 's. Networks $N_{5}$ and $N_{5}^{\prime}$ are attractor divergences. Network $N_{6}$ may either diverge or converge to $\{B\}$. Network $N_{7}$ may converge to an infinity of observation sets $\{n B\}$ where $n \in \mathbb{N}_{0}$.

An attractor divergence is a solution that will change infinitely without looping. The typical example is an infinite growth of a chemical solution, which cannot be reversed by some degradation. For instance, the network $\langle\emptyset,\{\emptyset \rightarrow A\}\rangle$ is an attractor divergence, since $A$ cannot be degraded, so that one can never come back to previous solutions but always continue, i.e., no attractor is ever reached.

Example 3. We next illustrate the observation predicates at the series of reaction networks, whose accessiblity graphs are given in Fig. 1. For these, we use the set of species Spec $=\left\{A, B, C, C^{\prime}, D\right\}$ and the observation function $\Omega$ that counts the number of molecules for all species, except for $C$ and $C^{\prime}$. For the latter, it identifies $C$ with $C^{\prime}$ and observes only the existence of either of the two. More formally, this can be defined by $\Omega\left(n_{1} A+n_{2} B+n_{3} C+n_{4} C^{\prime}+n_{5} D\right)=$ $n_{1} A+n_{2} B+m C+n_{5} D$ where $m=\max \left(n_{3}+n_{4}, 1\right)$.

Network $N_{1}=\langle A,\{A \rightarrow B, A \rightarrow C\}\rangle$ may reach two attractors, $\{B\}$ and $\{C\}$. Hence $N_{1} \downarrow_{\{B\}}$ and $N_{1} \downarrow_{\{C\}}$. This network may not diverge, i.e., $\neg N_{1} \uparrow$, so it must converge to either $\{B\}$ or $\{C\}$.

Network $N_{2}=\left\langle A,\left\{A \rightarrow B, A \rightarrow C, C \leftrightarrow C^{\prime}\right\}\right\rangle$ has two attractors $\{B\}$ and $\left\{C, C^{\prime}\right\}$. Since the observation function identifies $C^{\prime}$ with $C, N_{2}$ may converge to 
the same sets of observables than $N_{1}$, i.e., $N_{2} \downarrow_{\{B\}}$ and $N_{2} \downarrow_{\{C\}}$. Furthermore, it may not diverge, so it must converge to either $\{B\}$ or $\{C\}$, equally to $N_{1}$.

Network $N_{3}=\langle A,\{A \rightarrow B, A \rightarrow C, C \leftrightarrow 2 C\}\rangle$ has the singleton attractor $\{B\}$ and the infinite attractor $\{n C \mid n>0\}$. Since the observation function tests only the presence of $C$ 's, once again, $p_{4}$ must converge to either $\{B\}$ or $\{C\}$, equally to $N_{1}$ and $N_{2}$.

Network $N_{4}=\langle A,\{A \rightarrow A, A \rightarrow B, A \rightarrow C\}\rangle$ has an infinite reduction but may not reach an attractor divergence, and thus may not diverge. Such an infinite reduction is sometimes called a "weak divergence". It is raised by unfairness, if only the same reaction $A \rightarrow A$ is applied, and never any of the other two applicable reactions. Otherwise, $N_{4}$ can reach the attractors $\{B\}$ or $\{C\}$ and no other attractors. Therefore it must converge to $\{B\}$ or $\{C\}$, equally to $N_{1}, N_{2}$, and $N_{3}$.

Networks $N_{5}=\langle\emptyset,\{\emptyset \rightarrow A\}\rangle$ et $N_{5}^{\prime}=\langle\emptyset,\{\emptyset \rightarrow B\}\rangle$ are both attractor divergences, so that they may not converge.

Network $N_{6}=\langle A,\{A \rightarrow B, A \rightarrow C, C \rightarrow 2 C\}\rangle$ may converge to $\{B\}$ if we apply first the reaction $A \rightarrow B$, but may also diverge if we begin by the reduction with applying $A \rightarrow C$. This is an example where may divergence and may convergence are not exclusive.

Network $N_{7}=\langle A,\{\emptyset \stackrel{A}{\rightarrow} B, A \rightarrow \emptyset\}\rangle$ has an infinite sequence of reductions $A \rightarrow(A+B) \rightarrow(A+2 B) \ldots \rightarrow(A+n B) \ldots$, but may never diverge. This weak divergence is again raised by never applying the reaction $A \rightarrow \emptyset$ in an unfair manner. Nevertheless, $N_{7}$ may not diverge, since it may at any time point still converge to either of the $\{n B\}$ where $n \in \mathbb{N}_{0}$, i.e., $N_{7} \downarrow_{\{n B\}}$ for all $n$.

The fact that our observation predicates ignore weak divergences is natural for stochastic semantics as in applications to biological systems, since the probability of weak divergences will always be zero. In other contexts, weak divergences can be ignored for fair reduction strategies (see e.g. [29]). It should be noticed, however, that there is no canonical notion of fair reduction for reaction networks, so this argument doesn't apply here.

Definition 5. Given an observation $\Omega$, we say that two reaction networks $N_{1}$ and $N_{2}$ are context-less attractor equivalent, denoted by $N_{1} \sim_{\Omega} N_{2}$, if for all $O \subseteq \Omega(S o l): N_{1} \downarrow O$ iff $N_{2} \downarrow O$ and $N_{1} \uparrow$ iff $N_{2} \uparrow$.

Example 4. With one molecule of Dox in the initial solution, the simplified Tet-On simple network $\left\langle D o x,\left\{\emptyset \stackrel{D o x}{\longrightarrow} G F P_{a}, G F P_{a} \rightarrow \emptyset\right\}\right\rangle$ is context-less equivalent to $\left\langle D o x,\left\{\emptyset \rightarrow G F P_{a}, G F P_{a} \rightarrow \emptyset\right\}\right\rangle$ with respect to $\Omega_{\text {Tet-On }}$, since they both of them must converge to $\mathbb{N}_{0}$. This means that they may converge to $\mathbb{N}_{0}$, they may not converge to any other set of observations, and they do not diverge.

Example 5. We reconsider the reaction networks from Fig. 1. The networks $N_{1}$, $N_{2}, N_{3}$, and $N_{4}$ are context-less equivalent with respect to the observation function chosen in Example 3 since all of them must converge to $\{B\}$ or $\{C\}$, but may not be context-less equivalent for other observation functions. Note that number of times in which the same observations can be reached does not matter. 
Network $N_{5}$ and $N_{5}^{\prime}$ are context-less attractor equivalent even though they use different species. More generally, all attractor divergent solutions are contextless attractor equivalent.

Network $N_{6}$ is not context-less attractor equivalent to the others, since it may diverge and converge.

Network $N_{7}$ is the only one that may converge to infinitely many observation sets, so it is not context-less attractor equivalent to any other.

Let us emphasize the fact that the observation predicates $\downarrow_{O}$ and $\uparrow$ are sufficient to reason about other convergence and divergence predicates. For instance, a network must converge iff it cannot diverge. A network must converge to $O$ iff it may neither diverge nor converge to any other set of observations. And a network must diverge iff it may not converge to any $O$ (such as attractor divergences). So adding these three alternative observation predicates would not change our notion of context-less attractor equivalence.

\section{Attractor equivalence}

We next make our attractor equivalence context dependent. The chosen class of admissible contexts should capture what kind of inputs an experimental platform can make on an biological system.

A context is a reaction network. The application of a context $\mathcal{C}=\langle s, R\rangle$ to a network $N=\left\langle s^{\prime}, R^{\prime}\right\rangle$ is defined by the union of their solutions and reactions:

$$
\mathcal{C}[N]=\left\langle s^{\prime}+s, R^{\prime} \cup R\right\rangle
$$

However, for a given biological experiment, not all contexts are admissible. Indeed, a context may not have access to some biological species because of compartmentalization. Furthermore, some simplifications of a reaction networks may only be correct only if restricting the set of admissible contexts. There are many different manners to restrict the set of contexts to a subclass of admissible contexts. Here, we adopt the simplest method, which is to fix a set $\mathcal{I} \subseteq$ Spec of input species that can be touched by the context.

Definition 6. A context $\langle s, R\rangle$ is admissible for $\mathcal{I}$ if all species $A$ with $A \in s$ or $A \in R$ belong to in $\mathcal{I}$.

Definition 7 (Attractor equivalence). We call two reaction networks $N_{1}$ and $N_{2}$ attractor equivalent and write $N_{1} \equiv \equiv_{\Omega, \mathcal{I}} N_{2}$ iff for any context $\mathcal{C}$ admissible for $\mathcal{I}$ satisfies the context-less attractor equivalence $\mathcal{C}\left[N_{1}\right] \sim_{\Omega} \mathcal{C}\left[N_{2}\right]$.

Example 6. In the Tet-On system, the only input species that can be added by the microfluedic device is $D o x$, so we define $\mathcal{I}_{\text {Tet-On }}=\{D o x\}$. The simplified Tet-On network $N_{1}=\left\langle D o x,\left\{\emptyset \stackrel{D o x}{\longrightarrow} G F P_{a}, G F P_{a} \rightarrow \emptyset\right\}\right\rangle$ is context-less attractor equivalent to $N_{2}=\left\langle D o x,\left\{\emptyset \rightarrow G F P_{a}, G F P_{a} \rightarrow \emptyset\right\}\right\rangle$ but not attractor equivalent, since $\mathcal{C}=\langle\emptyset,\{D o x \rightarrow \emptyset\}\rangle$ is admissible, but $\mathcal{C}\left[N_{1}\right]$ must converge to $\{0\}$, while $\mathcal{C}\left[N_{2}\right]$ must converge to $\mathbb{N}_{0}$. 


$$
\begin{aligned}
& \frac{A \notin s \quad \Omega(A)=\Omega(s) \quad A \notin \mathcal{I}}{\left\langle s_{0}, R \cup\{s \leftrightarrow A\}\right\rangle \equiv_{\Omega, \mathcal{I}}^{R e v}\left\langle s_{0}[s / A], R[s / A]\right\rangle} \text { (REversible) } \\
& \frac{s_{0}(A) \geq n \quad A \notin s_{1} \quad \forall r \in R . p r_{r}(A) \geq 0 \quad A \notin \mathcal{I}}{\left\langle s_{0}, R \cup\left\{s_{1} \stackrel{n A}{\longrightarrow} s_{2}\right\}\right\rangle \equiv_{\Omega, \mathcal{I}}^{E n z}\left\langle s_{0}, R \cup\left\{s_{1} \rightarrow s_{2}\right\}\right\rangle}(\text { ENZYME) } \\
& \frac{\forall s_{1} \rightarrow s_{2} \in R . A \notin s_{1} \quad \Omega(A)=\mathbf{0} \quad A \notin \mathcal{I}}{\left\langle s_{0}+n A, R\right\rangle \equiv_{\Omega, \mathcal{I}}^{U s e}\left\langle s_{0}, R\right\rangle} \text { (UsELESs) } \\
& \frac{A \notin s_{0}+s+s^{\prime} \quad A \notin R \quad \Omega(A)=\mathbf{0} \quad A \notin \mathcal{I}}{\left\langle s_{0}, R \cup\left\{\emptyset \stackrel{s}{\rightarrow} A, A \rightarrow \emptyset, \emptyset \stackrel{A}{\rightarrow} s^{\prime}\right\}\right\rangle \equiv_{\Omega, \mathcal{I}}^{C a s 1}\left\langle s_{0}, R \cup\left\{\emptyset \stackrel{s}{\rightarrow} s^{\prime}\right\}\right\rangle}\left(\mathrm{CASCADE}_{1}\right) \\
& \frac{A \notin s_{0}+s+s^{\prime} \quad A \notin R \quad \Omega(A)=\mathbf{0} \quad A \notin \mathcal{I}}{\left\langle s_{0}, R \cup\left\{\emptyset \stackrel{s}{\rightarrow} A, A \rightarrow \emptyset, A \rightarrow s^{\prime}\right\}\right\rangle \equiv_{\Omega, \mathcal{I}}^{C a s 2}\left\langle s_{0}, R \cup\left\{\emptyset \stackrel{s}{\rightarrow} s^{\prime}\right\}\right\rangle}\left(\mathrm{CASCADE}_{2}\right)
\end{aligned}
$$

Fig. 2: Axioms of the attractor equivalence.

Example \%. We reconsider the examples in Fig. 1 with all species as input species $\mathcal{I}_{1}=\left\{A, B, C, C^{\prime}, D\right\}$. Networks $N_{1}$ and $N_{2}$ are then not attractor equivalent, since in the admissible context $\mathcal{C}=\left\langle\emptyset,\left\{C^{\prime} \rightarrow D\right\}\right\rangle, N_{2}$ may converge to $\{D\}$ in contrast to $N_{1}$. The context can thus make the difference between $C$ and $C^{\prime}$ visible, which is otherwise nonobservable. For the input species $\mathcal{I}_{2}=\{A, B, D\}$, the networks $N_{1}$ and $N_{2}$ are indeed attractor equivalent.

The networks $N_{1}$ and $N_{4}$ are attractor equivalent for any set of input species, since the context cannot distinguish either how may times the same set of observations is produced by an attractor.

Contexts can be used to cure or distinguish attractor divergences. For instance, with $A \in \mathcal{I}$, and $\mathcal{C}=\langle\emptyset,\{A \rightarrow \emptyset\}\rangle$, the network $\mathcal{C}\left[N_{5}\right]$ can converge to $\left\{n A \mid n \in \mathbb{N}_{0}\right\}$. And so it is not equivalent to $N_{5}^{\prime}=\langle\emptyset,\{\emptyset \rightarrow B\}\rangle$, since $\mathcal{C}\left[N_{5}^{\prime}\right]$ must diverge.

Example 8. If the set of input species contains "intermediate" species of a reaction cascade, then context may be used to make them visible. For instance, the networks $\langle A,\{A \rightarrow B\}\rangle$ and $\langle A,\{A \rightarrow C, C \rightarrow B\}\rangle$ are not equivalent if $C$ is an input species. They can then be distiguished by the context $\langle\emptyset,\{C \rightarrow D\}\rangle)$

\section{Axioms of attractor equivalence}

We present 5 axioms of attractor equivalence that we will use in the next section to simplification of the Tet-On reaction networks from [20].

The axioms for the attractor equivalence $\equiv_{\Omega, \mathcal{I}}$ are given in Fig. 2. There we use the symbol $\mathbf{0}$ for the zero of the observations monoid to which $\Omega$ is mapping. 
Axiom (REVERSIBLE) removes a reversible reaction between a molecule $A$ and a solution $s$, and substitutes $A$ with $s$ (denoted $[s / A]$ ) everywhere in the network. In order to do that, $A$ and $s$ must have the same observations, $A$ should not occur in $s$, and $A$ should not be modifiable by the context, that is: $\Omega(A)=\Omega(s), A \notin s$, and $A \notin \mathcal{I}$. For instance, this axiom can be used to simplify an enzymatic reaction network as below in Michaelis-Menten style:

$$
\langle\emptyset,\{(E+S) \leftrightarrow C, C \rightarrow(E+P)\}\rangle
$$

Here, an enzyme $E$ must bind to substrate $S$ forming a complex $C$, in order to produce product $P$ from $S$ while freeing $E$. The assumptions for applying (REVERSIBLE) are that an admissible context cannot modify the complex $C$ and that the complex produces the same observations than $E+S$. The above network can then be simplified to the equivalent network:

$$
\langle\emptyset,\{S \stackrel{E}{\rightarrow} P\}\rangle
$$

Axiom (ENZYME) removes an enzyme from a reaction, if it must always be present in sufficient amounts to activate the reaction, that is, if there is enough enzyme in the initial solution and if the enzyme cannot be degraded. Axiom (USELESS) removes from the initial solution all molecules of a non observable species that are never consumed by any reaction. The axioms $\left(\mathrm{CASCADE}_{1}\right)$ and $\left(\mathrm{CASCADE}_{2}\right)$ reduce an enzymatic cascade that, from a solution $s$, produces a solution $s^{\prime}$ mediated by a degradable molecule $A$. A molecule $A$ can only be used in three reactions: a production reaction, with enzymes $s(\emptyset \stackrel{s}{\rightarrow} A)$, a degradation reaction $(A \rightarrow \emptyset)$, and a reaction $\left(\emptyset \stackrel{A}{\rightarrow} s^{\prime}\right.$ or $\left.A \rightarrow s^{\prime}\right)$ that produces some $s^{\prime}$ provided that a molecule $A$ is present in the solution. Moreover, $A$ is not observable, and not modifiable by the context. Then we can replace the three previous reactions by the direct production of $s^{\prime}$ from $s$, that is $\emptyset \stackrel{s}{\rightarrow} s^{\prime}$.

The following theorem states that all these axioms are sound with respect to the attractor equivalence.

Theorem 1 (Soundness). For any $X \in\left\{R e v, E n z, U s e, \mathrm{Cas}_{1}, \mathrm{Cas}_{2}\right\}$,

$$
\equiv_{\Omega, \mathcal{I}}^{X} \subseteq \equiv_{\Omega, \mathcal{I}}
$$

Proof. We only show here the rather basic proof for axiom (USELESS) and the more complicated case of $\left(\mathrm{CASCADE}_{1}\right)$. The proof of the other three axioms is similar and can be found in Appendix A. Remember that a set $S$ is an attractor for $N$ iff it is closed under reduction and strongly connected, and that a network may diverge iff an attractor divergence is reachable.

Let $N \equiv{ }_{\Omega, \mathcal{I}}^{U \text { se }} N^{\prime}$ and $\mathcal{C}$ be an admissible context with respect to $\mathcal{I}$. Let $N=$ $\left\langle s_{0}, R \cup\left\{s_{1} \stackrel{n A}{\longrightarrow} s_{2}\right\}\right\rangle$ and $N^{\prime}=\left\langle s_{0}, R \cup\left\{s_{1} \rightarrow s_{2}\right\}\right\rangle$. Since $A$ cannot belong to the reactants of any reaction in $R$ and $\mathcal{C}$, for any solution $s$ any reaction $r$ applicable in $s+n A$ is also applicable in $s$. Hence, for any solution $s$ and $s^{\prime}$ :

$$
(s+n A) \underset{\mathcal{C}[N]}{\rightarrow^{*}}\left(s^{\prime}+n A\right) \quad \text { iff } \quad s \underset{\mathcal{C}\left[N^{\prime}\right]}{\vec{x}^{*}} s^{\prime}
$$


Moreover, the number of $A$ in the solutions of both networks cannot decrease. So in particular, any reachable solution in $\mathcal{C}[N]$ contains at least $n A$. Let $S$ be an attractor reachable from $\mathcal{C}[N]$, it must be of the form $S=\left\{\left(s^{\prime}+n A\right) \mid s^{\prime} \in S o l\right\}$. Then the set $S^{\prime}=\left\{s^{\prime} \mid\left(s^{\prime}+n A\right) \in S\right\}$ is an attractor reachable from $\mathcal{C}\left[N^{\prime}\right]$. Reversely, for any attractor $S^{\prime}$ reachable from $\mathcal{C}\left[N^{\prime}\right]$, the set $S=\left\{\left(s^{\prime}+n A\right) \mid\right.$ $\left.s^{\prime} \in S^{\prime}\right\}$ is an attractor reachable from $\mathcal{C}[N]$.

The assumption of the axiom $\Omega(A)=\mathbf{0}$ implies $\Omega(S)=\Omega\left(S^{\prime}\right)$. Hence for any $O, \mathcal{C}[N]$ may converge to $O$ iff $\mathcal{C}\left[N^{\prime}\right]$ may converge to $O$.

Moreover, if a solution $s+n A$ must diverge and is reachable from $\mathcal{C}[N]$, then $s$ must diverge and is reachable from $\mathcal{C}\left[N^{\prime}\right]$, and reversely. So $\mathcal{C}[N]$ may diverge iff $\mathcal{C}\left[N^{\prime}\right]$ may diverge, and so $N$ and $N^{\prime}$ are attractor equivalent, that is $N \equiv_{\Omega, \mathcal{I}} N^{\prime}$.

We next consider axiom $\left(\mathrm{CASCADE}_{1}\right)$. We chose an instance $N \equiv_{\Omega, \mathcal{I}}^{C a s 1} N^{\prime}$ of this axiom with some networks $N=\left\langle s_{0}, R \cup\left\{\emptyset \stackrel{s}{\rightarrow} A, A \rightarrow \emptyset, \emptyset \stackrel{A}{\rightarrow} s^{\prime}\right\}\right\rangle$ and $N^{\prime}=\left\langle s_{0}, R \cup\left\{\emptyset \stackrel{s}{\rightarrow} s^{\prime}\right\}\right\rangle$, and an context $\mathcal{C}$ that is admissible for $\mathcal{I}$ and thus does not contain $A$ molecules. For any solution $s_{1}$ and $s_{2}$ that do not contain $A$ molecules, we will show that:

$$
s_{1} \overrightarrow{\mathcal{C}[N]}^{*} s_{2} \quad \text { iff } \quad s_{1}{\overrightarrow{\mathcal{C}\left[N^{\prime}\right]}}^{*} s_{2}
$$

For the implication from left to right, we will first do the following transformations on the sequence of reduction steps justifying $s_{1} \underset{\mathcal{C}[N]}{{ }^{*}} s_{2}$. Since $A \notin s_{1}$, $A \notin R$ and $A \notin \mathcal{I}$, we can show that any step with $\emptyset \stackrel{A}{\rightarrow} s^{\prime}$ must be preceded by a step with $\emptyset \stackrel{s}{\rightarrow} A$ but not necessarily immediately. The first transformation will move them directly after the step with $\emptyset \stackrel{s}{\rightarrow} A$. The resulting reduction sequence is still a valid for $\mathcal{C}[N]$, since $A$ cannot be used in other reactions. We will also move all steps with $A \rightarrow \emptyset$ after the previous $A \rightarrow s^{\prime}$. So the transition sequence can be decomposed into the following parts: first a part without $A$, then the production of $A$ by $s$, some productions of $s^{\prime}$ with $A$ as an enzyme, and possibly degradations of $A$, then again a part without $A$, etc. We can then imitate the transition into $\mathcal{C}\left[p^{\prime}\right]$, by replacing the parts with $A$ by $\emptyset \stackrel{s}{\rightarrow} s^{\prime}$. For the implication from the right to the left, it is sufficient to start with a reduction sequence for $s_{1}{\overrightarrow{\mathcal{C}\left[N^{\prime}\right]}}^{*} s_{2}$, and to replace any step $\emptyset \stackrel{s}{\rightarrow} s^{\prime}$ by three subsequent steps with $\emptyset \stackrel{s}{\rightarrow} A, \emptyset \stackrel{A}{\rightarrow} s^{\prime}$, and $\left.A \rightarrow \emptyset\right)$. We will next prove the following for all sets of observations $O$ that:

$$
\mathcal{C}[N] \downarrow_{O} \text { iff } \mathcal{C}\left[N^{\prime}\right] \downarrow_{O} .
$$

Let $S$ be an attractor reachable from $\mathcal{C}[N]$ and $s_{1} \in S$. Then $s_{1}^{\prime}=s_{1} \backslash A$ is also in $S$, since it can be obtained by applying reaction $A \rightarrow \emptyset$ repeatedly. Let $S^{\prime}=\operatorname{Acc}_{\mathcal{C}\left[N^{\prime}\right]}\left(s_{1}^{\prime}\right)$. We note that $S^{\prime}$ is closed under reduction and reachable from $\mathcal{C}\left[N^{\prime}\right]$. In order to show that $S^{\prime}$ is strongly connected, we chose $s_{2}^{\prime} \in S^{\prime}$ and show how to reach $s_{1}^{\prime}$ from $s_{2}^{\prime}$. Since $s_{2}^{\prime} \in S$, we have $s_{1}^{\prime} \underset{\mathcal{C}\left[N^{\prime}\right]}{{ }^{*}} s_{2}^{\prime}$. Hence $s_{1}^{\prime} \underset{\mathcal{C}[N]}{\vec{C}^{*}} s_{2}^{\prime}$ by the above claim (and since $A \notin s_{1}^{\prime}$, and $A \notin s_{2}^{\prime}$ ), so that $s_{2}^{\prime}$ and $s_{1}^{\prime}$ belong to 
attractor $S$ for the reactions of $\mathcal{C}[N]$, so that we can conclude $s_{2}^{\prime} \underset{\mathcal{C}[N]}{\vec{N}^{*}} s_{1}^{\prime}$ and $s_{2}^{\prime} \underset{\mathcal{C}\left[N^{\prime}\right]}{\vec{x}^{*}} s_{1}^{\prime}$. Hence, $S^{\prime}$ is an attractor reachable from $\mathcal{C}\left[N^{\prime}\right]$. Since $\Omega(A)=\emptyset$, we directly have $\Omega(S)=\Omega\left(S^{\prime}\right)$. So if $\mathcal{C}[N] \downarrow_{\Omega(S)}$ then $\mathcal{C}\left[N^{\prime}\right] \downarrow_{\Omega\left(S^{\prime}\right)}$ and thus $\mathcal{C}\left[N^{\prime}\right] \downarrow_{\Omega(S)}$.

Conversely, let $S^{\prime}$ be a reachable attractor for $\mathcal{C}\left[N^{\prime}\right]$, and $s_{1}^{\prime} \in S^{\prime}$. Then $s_{1}^{\prime}$ is also reachable in $\mathcal{C}[N]$. Let $S=\operatorname{Acc}_{\mathcal{C}[N]}\left(s_{1}^{\prime}\right)$. It is closed under reduction and reachable. Let $s_{2} \in S$, i.e., $s_{1}^{\prime} \underset{\mathcal{C}[N]}{\rightarrow^{*}} s_{2}$. Then we can apply $A \rightarrow \emptyset$ and do $s_{2} \underset{\mathcal{C}[N]}{\vec{C}}{ }^{*} s_{2}^{\prime}$ with $s_{2}^{\prime}=s_{2} \backslash A$. So $s_{1}^{\prime} \underset{\mathcal{C}[N]}{\vec{C}}{ }^{*} s_{2}^{\prime}$ implies $s_{1}^{\prime} \underset{\mathcal{C}\left[N^{\prime}\right]}{*} s_{2}^{\prime}$, so $s_{2}^{\prime} \in S^{\prime}$, and we can do $s_{2}^{\prime} \underset{\mathcal{C}\left[N^{\prime}\right]}{*} s_{1}^{\prime}$ and $s_{2}^{\prime} \underset{\mathcal{C}[N]}{\rightarrow^{*}} s_{1}^{\prime}$. So we can also do $s_{2} \underset{\mathcal{C}[N]}{\rightarrow^{*}} s_{1}^{\prime}$, so $S$ is strongly connected and is an attractor. So if $\mathcal{C}\left[N^{\prime}\right] \downarrow_{\Omega\left(S^{\prime}\right)}$ then $\mathcal{C}[N] \downarrow_{\Omega(S)}$ and since $\Omega(S)=\Omega\left(S^{\prime}\right)$, we have $\mathcal{C}[N] \downarrow_{\Omega\left(S^{\prime}\right)}$. It remains to show that:

$$
\mathcal{C}[N] \uparrow \operatorname{iff} \mathcal{C}\left[N^{\prime}\right] \uparrow
$$

If there an attractor divergence $s_{1}$ reachable from $\mathcal{C}[N]$, then $s_{1} \backslash A$ must also diverge in $\mathcal{C}[N]$, and it is reachable and must diverge in $\mathcal{C}\left[N^{\prime}\right]$ too. Conversely, if $s_{1}^{\prime}$ is an attractor divergence reachable from $\mathcal{C}\left[N^{\prime}\right]$, then it is directly reachable in $\mathcal{C}[N]$, and must diverge too. In summary, $\mathcal{C}[N]$ and $\mathcal{C}\left[N^{\prime}\right]$ are context-less attractor equivalent for all admissible contexts $\mathcal{C}$, so that $N \equiv_{\Omega, \mathcal{I}} N^{\prime}$.

\section{Simplification of detailed Tet-On network}

We now show how to use the axioms of the attractor equivalence for simplying the network of a concrete biological system. We have chosen the Tet-On system $[20,14,15]$ that is illustrated in Fig. 3. While still being rather simple compared to other networks in biological applications, it can already be used to illustrate the power of the attractor equivalence.

The Tet-On system describes how the producton of activated green fluorescent proteins $\left(G F P_{a}\right)$ in a cell can be stimulated by the presence of doxycycline (Dox) outside the cell. The detailed reaction network from [20] is:

$$
\text { Tet-Ondetailed }=\left\langle 5 P_{\text {TRE3G }}+45000 r t T A, R^{(0)}\right\rangle
$$

where $R^{(0)}$ is the set of reactions (2-10) from Fig. 4. The initial solution contains 5 copies of the gene $P_{T R E 3 G}$ and 45000 molecules $r t T A$ (i.e. a concentration of about $1.1 \mu \mathrm{g} / \mathrm{mL})$.

The enviroment of the cell is controlled by a micro-fluedic device, that is able to add Dox to the system. Dox can then move into the cell and become $D o x_{i}$ by reaction (2), where it can either be degraded by reaction (3), or bind to the artificial transcription factor $r t T A$ by reaction (4). The complex $r t T A D o x$ can then either dissociate (4), or activate the transcription of the gene $P_{T R E 3 G}$, producing $m R N A$ (5). $m R N A$ can either be degraded (6) or be translated into GFP (7). Finally, GFP needs to be actived into $G F P_{a}$ in order to become 


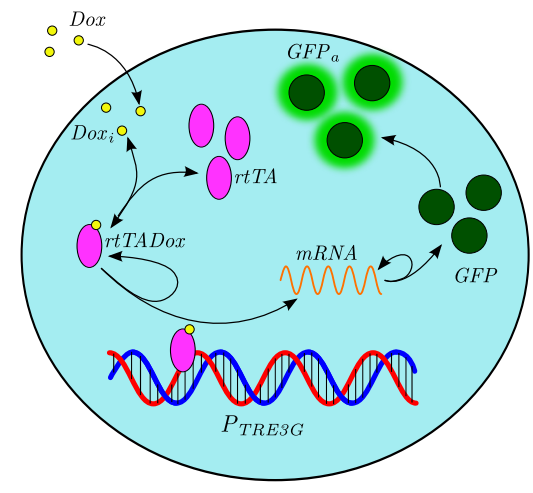

Fig. 3: Illustration of the detailed Tet-On network.

$$
\begin{array}{lll}
\emptyset \stackrel{\text { Dox }}{\longrightarrow} \text { Dox }_{i} & (2) & m R N A \rightarrow \emptyset \\
\text { Dox }_{i} \rightarrow \emptyset & (3) & \emptyset \stackrel{m R N A}{\longrightarrow} \text { GFP } \\
\left(r t T A+\text { Dox }_{i}\right) \leftrightarrow r t T A D o x & (4) & G F P \rightarrow G F P_{a} \\
\emptyset \stackrel{P_{T R E 3 G}+r t T A D o x}{\longrightarrow} m R N A & (5) & G F P \rightarrow \emptyset \\
& & G F P_{a} \rightarrow \emptyset
\end{array}
$$

Fig. 4: Reactions of network Tet-On detailed .

fluorescent and thus observable by a microscope (8). Both GFP and $G F P_{a}$ can also be degraded $(9,10)$.

We want to simplify the complex network Tet-On detailed to the simplified network Tet-On simple in (1), while consider the observation function $\Omega_{\text {Tet-On }}$ defined by $\Omega_{\text {Tet-On }}\left(m G F P_{a}+s\right)=m$ where $s$ is an arbitrary solution with $G F P_{a} \notin s$ and $m \in \mathbb{N}_{0}$, and with the set of input species $\mathcal{I}_{\text {Tet-On }}=\{D o x\}$.

By using the axioms from Fig. 2, we will show that the networks Tet-On detailed and Tet-On simple $_{\text {are }}$ attractor equivalent. We start with the complex network Tet-Ondetailed and simplify it by applying the axioms. Let us first consider the reversible reaction (4). Since $\Omega_{\text {Tet-on }}\left(r t T A+D o x_{i}\right)=\Omega_{\text {Tet-On }}(r t T A D o x)=0$ and $r t T A D o x \notin \mathcal{I}_{\text {Tet-On }}$, we can substitute $r t T A+D_{o x}$ for rtTADox by applying axiom (REVERSIBLE). This shows that Tet-Ondetailed is equivalent to the network:

$$
\text { Tet-On }^{(1)}=\left\langle 5 P_{\text {TRE3G }}+45000 r t T A, R^{(1)}\right\rangle
$$

where $R^{(1)}$ is obtained from the Tet-Ondetailed reaction set $R^{(0)}$ by removing reaction (4) and by replacing reaction (5) by:

$$
\emptyset \stackrel{P_{T R E 3 G}+r t T A+D_{o x}}{\longrightarrow} m R N A
$$


This reaction is then the only one that use $r t T A$. Since it is as an enzyme, and since $r t T A$ is present in sufficient amount in the initial solution, we can apply the axiom (ENZYME), and rtTA can be removed from that reaction. Similarly, we can remove $P_{T R E 3 G}$. So the reaction becomes:

$$
\emptyset \stackrel{D o x_{i}}{\longrightarrow} m R N A
$$

Then $P_{T R E 3 G}$ and $r t T A$ become useless, and since they are unobservable, they can be removed from the initial solution using the axiom (USELESS). Network Tet-On ${ }^{(1)}$ is thus equivalent to network: Tet-On ${ }^{(2)}=\left\langle\emptyset, R^{(2)}\right\rangle$ where $R^{(2)}$ contains the reactions $(2),(3),\left(5^{\prime \prime}\right)$, and the reactions from $(6-10)$. Now, $m R N A$ is unobservable, degradable, only producible by an enzymatic reaction $\left(5^{\prime \prime}\right)$, and is only used as an enzyme to produce GFP (7). Thus, we can remove it using axiom $\left(\mathrm{CASCADE}_{1}\right)$. We can then apply the axiom $\left(\mathrm{CASCADE}_{2}\right)$ to remove GFP, since it is also in an enzymatic cascade. So Tet-On ${ }^{(2)}$ is equivalent to the network Tet-On ${ }^{(3)}=\left\langle\emptyset, R^{(3)}\right\rangle$ where $R^{(3)}$ is the set of reactions (2), (3), ( $\left.8^{\prime}\right)$ and (10) and:

$$
\emptyset \stackrel{\operatorname{Dox}_{i}}{\longrightarrow} \operatorname{GFP}_{a}
$$

Finally, we can again apply $\left(\mathrm{CASCADE}_{1}\right)$ to remove $D o x_{i}$ which makes Tet-On ${ }^{(3)}$

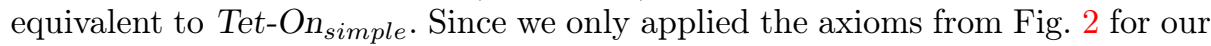
simplifications, Theorem 1 proves that indeed:

$$
\text { Tet-On } n_{\text {detailed }} \equiv_{\Omega_{\text {Tet-On }}, \mathcal{I}_{\text {Tet-On }}} \text { Tet-On } \text { simple }
$$

\section{Bisimulation and trace equivalences}

We now discuss alternative equivalences for reaction networks. These are based on bisimulations or traces of programs in some programming language, and can be transferred to reaction networks without particular difficulties, simply by considering reaction networks as programs.

We start with program equivalences based on bisimulations of programs, as developed for comparing concurrent programs in various calculi or programming languages $[25,30,38]$.

In the case of reaction networks, we assume an observation function $\Omega$ and a set of input species $\mathcal{I}$. A reduction $s \rightarrow s^{\prime}$ of a reaction network is silent if $\Omega(s)=\Omega\left(s^{\prime}\right)$. A silent action of a reaction network is a composition of finitely many silent reductions. An observable action is a composition of a silent action, a nonsilent reduction, and another silent action.

A (weak) bisimulation is a relation $R$ on programs such that any two bisimilar programs have the same observation with $\Omega$ and can do the same observable actions while remaining in $R$. Two programs are bisimilarity equivalent if they are related by some bisimulation relation in any admissible context. The axioms (REVERSIBLE), (ENZYME) and (USELESS) are sound for the bisimilation equivalence. This doesn't hold for $\left(\mathrm{CASCADE}_{1}\right)$ and $\left(\mathrm{CASCADE}_{2}\right)$ though, as we will argue below. Hence, simplifications with these two axioms cannot be justified 


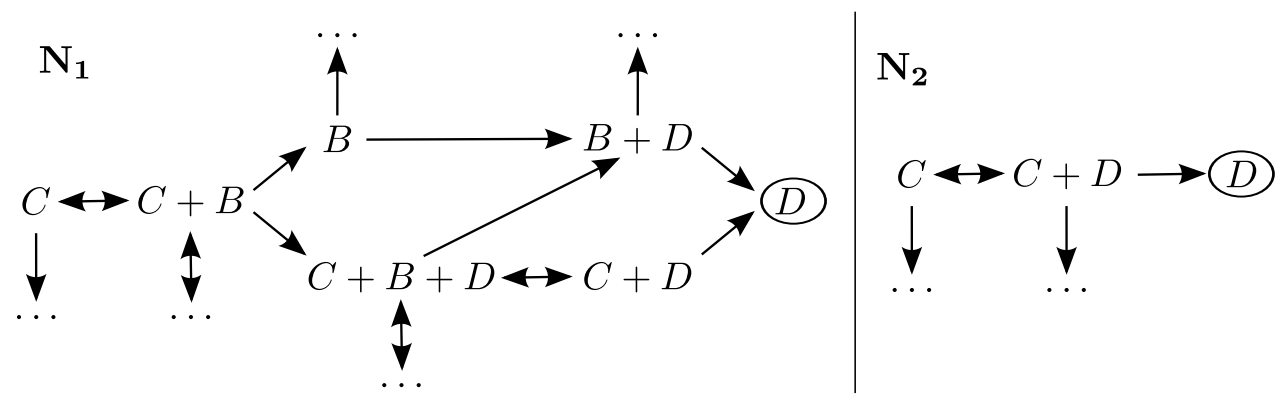

Fig. 5: $N_{1}$ and $N_{2}$ are attractor equivalent (using axiom ( $\mathrm{CASCADE}_{1}$ )), but nor bisimilarity equivalent@neither trace equivalent.

by the bisimilation equivalence, in contrast to the attractor equivalence as we showed in Theorem 1.

The argument is similar for both axioms. Consider for instance $N_{1} \equiv_{\Omega, \mathcal{I}}^{C a s 1} N_{2}$ of $\left(\mathrm{CASCADE}_{1}\right)$ with the networks following networks $N_{1}$ and $N_{2}$ whose reachability graphs are illustrated in Fig. 5:

$$
\begin{aligned}
& N_{1}=\langle C,\{C \rightarrow \emptyset, \emptyset \stackrel{C}{\rightarrow} B, B \rightarrow \emptyset, \emptyset \stackrel{B}{\rightarrow} D\}\rangle \\
& N_{2}=\langle C,\{C \rightarrow \emptyset, \emptyset \stackrel{C}{\rightarrow} D\}\rangle
\end{aligned}
$$

We choose the observation function $\Omega(s+n C+n D)=n C+m D$ where $C, D \notin s$ and for simplicity $\mathcal{I}=\emptyset$ so that only the empty context is admissible. The networks $N_{1}$ and $N_{2}$ are then attractor equivalent, since they both must converge to any $\{m D\}$ in all admissible contexts (since $B$ and $C$ can always be degraded but not $D$ ).

We next show that $N_{1}$ and $N_{2}$ are not bisimilarity equivalent. The problem is that one can reach from $N_{1}$ the nonobservable solution $B$, on which we can do an observable action to create observation $D$. In $N_{2}$, however, we need to reach $C$ for the creation of observation $D$. The problem is now that $B$ and $C$ cannot be in any bisimilarity relation since $\Omega(B) \neq \Omega(C)$, so $B$ cannot be in any bisimilarity relation to any solution of $N_{2}$.

Conversely, bisimilarity equivalence is not sufficient either to prove attractor equivalence. The problem is that the bisimilarity equivalence cannot distinguish some attractor divergences from some attractor convergences, when nonobservable molecules are concerned. For instance, consider the two networks in Fig. 6:

$$
\begin{aligned}
& N_{3}=\langle\emptyset,\{\emptyset \rightarrow B\}\rangle \\
& N_{4}=\langle\emptyset,\{\emptyset \rightarrow B, B \rightarrow \emptyset\}\rangle
\end{aligned}
$$

Since $B$ is nonobservable, $N_{3}$ and $N_{4}$ are in the bisimilarity equivalence. But $N_{3}$ is an attractor divergence, while $N_{4}$ may reach the attractor $\left\{n B \mid n \in \mathbb{N}_{0}\right\}$. 

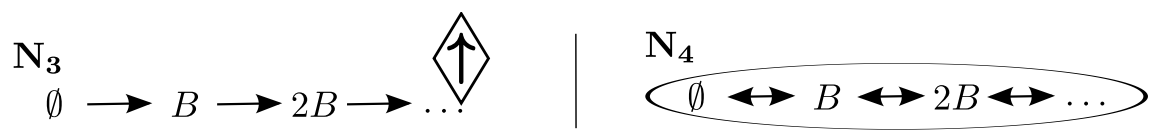

Fig. 6: $N_{3}$ and $N_{4}$ are (weak)-bisimilar, but not attractor equivalent.
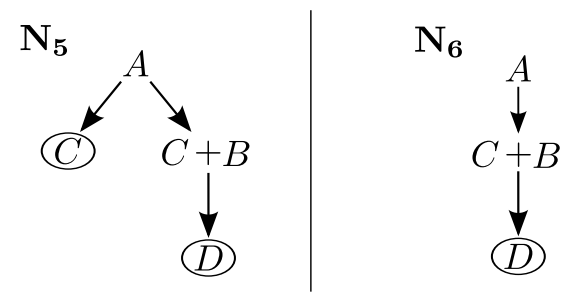

Fig. 7: $N_{5}$ and $N_{6}$ are trace equivalent, but not attractor equivalent.

Trace equivalences [18] offer an alternative to bisimilarity equivalences. A trace is a sequence (finite or not) of solutions $s_{i}$, s.t. there is a reduction from $s_{i}$ to $s_{i+1}$ for any $i$. A trace observation is a sequence of observations $\Omega\left(s_{i}\right)$, s.t. there is an observable action from $s_{i}$ to $s_{i+1}$. Two networks are trace equivalent if, in any admissible context, they have the same set of trace observations.

Once again the trace equivalence may detect differences in the intermediate behviour between input and output. In particular the axioms $\left(\mathrm{CASCADE}_{1}\right)$ and $\left(\mathrm{CASCADE}_{2}\right)$ will not preserve the trace equivalence. Note also that bisimilarity equivalence implies trace equivalence. Therefore, the axioms (REVERSIBLE), (ENZYME) and (USELESS) are also sound for the trace equivalence. Furthermore, the networks $N_{3}$ and $N_{4}$ from Fig. 6, which are not attractor equivalent, are also trace equivalent.

Beside of the problem that the trace equivalence can observe intermediate behaviors between input and output, an other problem is that it cannot detect termination or attractor convergences. But one would need to detect termination in order to distinguish the following two networks, whose reachability graph is illustrated in Fig. 7:

$$
\begin{aligned}
N_{5} & =\langle A,\{A \rightarrow C, A \rightarrow(C+B),(C+B) \rightarrow D\}\rangle \\
\text { and } N_{6} & =\langle A,\{A \rightarrow(C+B),(C+B) \rightarrow D\}\rangle
\end{aligned}
$$

Networks $N_{5}$ and $N_{6}$ have the same trace observations since $B$ is not observable, but they are not attractor equivalent, since $N_{5}$ may converge to $C$ or $D$, while $N_{6}$ may only converge to $D$.

One could hope to solve this problem with alternative trace equivalences that are based on termination, as considered by [13,21] for instance. But since most biological system reach a cyclic attractor rather than to terminate, a terminatedtrace equivalence would pose other important problems. For instance, we could 
slightly modify the above two networks by adding an extra loop between two fresh observable molecules, $E \leftrightarrow F$. Then the two networks would still have the same terminated-traces (none), and also the same infinite traces, but would fail to be attractor equivalent.

One could also hope to cure the notion of trace equivalence, so that it becomes included in the attractor equivalence, by restriction to some kind of fair traces. Unfortunately, it is difficult to find a good notion of fairness for Petri nets as shown in [19], were 24 different notions were proposed. Furthermore, the usual notions don't really match with what we need in systems biology or with other kinds of stochastic systems. For instance:

- if we take a fairness notion that no reaction can be ignored infinitely [19,34], then in the network $\langle A,\{A \rightarrow 2 A, A \rightarrow \emptyset\}\rangle$, the trace $A \rightarrow 2 A \rightarrow A \rightarrow 2 A \rightarrow$ $\ldots$ is fair. But this trace is a weak divergence, so it may appear as unfair, since it always reach the empty solution at any time point but never does.

- if we take as fairness notion that no infinitely reachable solution is infinitely ignored [19,34], then the network $\langle A,\{\emptyset \stackrel{A}{\rightarrow} I, A \rightarrow B\}\rangle$ has the fair the trace $A \rightarrow(A+I) \rightarrow(A+2 I) \rightarrow(A+3 I) \rightarrow \ldots$, while the second reaction is never used (even though it is always applicable).

- if we try to combine the two previous fairness notions, i.e., we can neither ignore any reactions nor any reachable solution, then in the network $\langle A,\{A \rightarrow 2 A, A \rightarrow B\}$,$\rangle , that must converge, the following divergent trace$ is fair $A \rightarrow 2 A \rightarrow(A+B) \rightarrow(2 A+B) \rightarrow(A+2 B) \rightarrow(2 A+2 B) \rightarrow \ldots$ (we use infinitely the 2 reactions, and the increase of $B$ implies that there is no infinitely reachable solution)

\section{Related work}

Program equivalences for sequential or concurrent programming languages are usually focused on the reachability of termination [39], which is too restrictive for reaction networks.

The notion of attractors also exists in logical regulatory networks [28,27]. These networks are quite similar to ours, but the number of each molecule is bounded, and so there are a finite number of reachable solutions, and their attractors are then finite. In [28] a simplification method for logical regulatory networks is proposed that preserves attractors, but new attractors may be created, so the reachability of the attractors may be changed. In [27], these methods are adapted so that they also preserve the reachability of attractors.

Other simplification methods have been proposed for biological systems. Plotkin [32] proposed a calculus of chemical systems, where networks are sets of reactions, without initial solution. He described then several contextual operational semantics, and some associated equivalences. In [12], the authors used subgraph epimorphisms in order to link different models. In [10], the authors used bisimulations for BioPEPA models. 
Since our reaction networks can actually be seen as Petri nets, it is also interesting to look after equivalences and simplifications of Petri Nets [33]. Berthelot $[3,2]$ has for instance proposed several simplification rules, that have been generalized for time Petri Nets [40] or for coloured Petri Nets [16]). But these rules are made to preserve usual Petri Nets properties (safety, boundedness, liveness, etc), and not the final behaviour. Other simplification methods $[47,44,24]$ also only focus on preserving liveness and boundedness. Murata et al. also introduce new simplifications $[26,23]$, but restricted to marked graphs, a subclass of Petri Nets. Simplifications also exist for free-choice nets, another subclass of Petri Nets $[6,8]$. Heiner et al. [17] also proposed analysis of biological systems represented by Petri Nets, but they are more focus on the transient behavior of the system.

Simplification methods also exist for models of biological systems, whose operational semantics accounts for time. For instance, in [7] they reduce systems of ordinary differential equations by merging some molecules, and Batmanov et al.[1] take advantage of symmetries to reduce a model while preserving stochastic properties. Other methods use difference between the speed of the reactions [35,41,11], or local equilibrium [43].

\section{Conclusion}

We presented the attractor equivalence for reaction networks without kinetic functions, and argued that it yields an appropriate semantics for reaction networks in systems biology. In particular we have shown that it supports powerful axioms that make it suitable for model simplification of concrete biological systems.

In future work, we plan to elaborate a more complete set of axioms, and to develop simplification algorithm that can be applied more systematically to biological systems. It would also be nice to have a support for the simplication of reaction networks in the SBML format. On the scientific side, one of the problems is to add kinetics to our observational semantics. Further problems are how to develop with attractor equivalences for language of biochemical reactions such as Kappa and React(C).

\section{References}

1. K. Batmanov, C. Kuttler, F. Lemaire, C. Lhoussaine, and C. Versari. Symmetrybased model reduction for approximate stochastic analysis. CMSB2012, 2012.

2. G. Berthelot. Checking properties of nets using transformations. Advances in Petri Nets 1985, 1986.

3. G. Berthelot and G. Roucairol. Reduction of petri-nets. In Mathematical Foundations of Computer Science 1976, LNCS. 1976.

4. N. Chabrier-Rivier, F. Fages, and S. Soliman. The biochemical abstract machine BIOCHAM. In CMSB 2004, volume 3082 of LNCS. Springer Verlag, 2005.

5. V. Danos and C. Laneve. Formal molecular biology. Theoretical Computer Science, $325(1), 2004$. 
6. J. Desel. Reduction and design of well-behaved concurrent systems. In CONCUR '90, volume 458 of $L N C S$. Springer Berlin Heidelberg, 1990.

7. A. Dokoumetzidis and L. Aarons. Proper lumping in systems biology models. 3(October 2007), 2009.

8. J. Esparza and M. Silva. Top-down synthesis of live and bounded free choice nets. Advances in Petri Nets 1991, 1991.

9. F. Fages and S. Soliman. Formal cell biology in biocham. In $S F M$, volume 5016 of LNCS. Springer, 2008.

10. V. Galpin and J. Hillston. Equivalence and discretisation in Bio-PEPA. Computational Methods in Systems Biology, 2009.

11. V. Galpin, J. Hillston, and F. Ciocchetta. A semi-quantitative equivalence for abstracting from fast reactions. Electronic Proceedings in Theoretical Computer Science, 67(CompMod), Sept. 2011.

12. S. Gay, S. Soliman, and F. Fages. A graphical method for reducing and relating models in systems biology. Bioinformatics (Oxford, England), 26(18), Sept. 2010.

13. R. J. v. Glabbeek. The linear time-branching time spectrum. In Proceedings of the Theories of Concurrency: Unification and Extension. Springer-Verlag, 1990.

14. M. Gossen and H. Bujard. Tight control of gene expression in mammalian cells by tetracycline-responsive promoters. Proceedings of the National Academy of Sciences of the United States of America, 89(12), June 1992.

15. M. Gossen, S. Freundlieb, G. Bender, G. Müller, W. Hillen, and H. Bujard. Transcriptional activation by tetracyclines in mammalian cells. Science (New York, N.Y.), 268(5218), June 1995.

16. S. Haddad. A reduction theory for coloured nets. High-level Petri Nets, 1991.

17. M. Heiner, D. Gilbert, and R. Donaldson. Petri nets for systems and synthetic biology. In Formal methods for computational systems biology. Springer, 2008.

18. C. Hoare. A model for communicating sequential process. 1980.

19. R. Howell, L. Rosier, and H. Yen. A taxonomy of fairness and temporal logic problems for Petri nets. Theoretical Computer Science, 82, 1991.

20. Z. Huang, C. Moya, A. Jayaraman, and J. Hahn. Using the Tet-On system to develop a procedure for extracting transcription factor activation dynamics. Molecular bioSystems, 6(10), Oct. 2010.

21. M. Jantzen. Language theory of Petri nets. Petri Nets: Central Models and Their Properties, 1987.

22. M. John, C. Lhoussaine, J. Niehren, and C. Versari. Biochemical reaction rules with constraints. In European Symposium on Programming Languages, LNCS, Saarbrücken Allemagne, 2011. Springer Verlag.

23. R. Johnsonbaugh and T. Murata. Additional methods for reduction and expansion of marked graphs. Circuits and Systems, IEEE Transactions on, 28(10), 1981.

24. K.-H. Lee and J. Favrel. Hierarchical reduction method for analysis and decomposition of Petri nets. IEEE Transactions on Systems, Man, and Cybernetics, SMC-15(2), Mar. 1985.

25. R. Milner. A Calculus of Communicating Systems, volume 92 of LNCS. Springer, 1980 .

26. T. Murata and J. Koh. Reduction and expansion of live and safe marked graphs. Circuits and Systems, IEEE Transactions on, 27(1), 1980.

27. A. Naldi, P. T. Monteiro, and C. Chaouiya. Efficient handling of large signallingregulatory networks by focusing on their core control. In Computational Methods in Systems Biology. Springer, 2012.

28. A. Naldi, E. Remy, D. Thieffry, and C. Chaouiya. Dynamically consistent reduction of logical regulatory graphs. Theoretical Computer Science, 412(21), May 2011. 
29. J. Niehren, D. Sabel, M. Schmidt-Schauß, and J. Schwinghammer. Observational semantics for a concurrent lambda calculus with reference cells and futures. In 23rd Conference on Mathematical Foundations of Programming Semantics, volume 173 of Electronical notes in theoretical computer science. Elsevier, Apr. 2007.

30. D. M. R. Park. Concurrency and automata on infinite sequences. In Theoretical Computer Science, volume 104 of LNCS. Springer, 1981.

31. A. M. Pitts. Operational semantics and program equivalence. In APPSEM, volume 2395 of LNCS. Springer, 2000.

32. G. D. Plotkin. A calculus of chemical systems. In In Search of Elegance in the Theory and Practice of Computation, volume 8000 of LNCS. Springer, 2013.

33. L. Pomello, G. Rozenberg, and C. Simone. A survey of equivalence notions for net based systems. Advances in Petri Nets 1992, 1992.

34. J. Queille and J. Sifakis. Fairness and related properties in transition systems - a temporal logic to deal with fairness. Acta Informatica, 220, 1983.

35. O. Radulescu, A. N. Gorban, A. Zinovyev, and A. Lilienbaum. Robust simplifications of multiscale biochemical networks. BMC systems biology, 2, Jan. 2008.

36. V. N. Reddy, M. L. Mavrovouniotis, and M. N. Liebman. Petri net representations in metabolic pathways. In ISMB. AAAI, 1993.

37. D. Sabel and M. S. Schauss. Conservative concurrency in haskell. In LICS. IEEE, 2012.

38. D. Sangiorgi, N. Kobayashi, and E. Sumii. Environmental bisimulations for higherorder languages. ACM Trans. Program. Lang. Syst., 33(1), Jan. 2011.

39. M. Schmidt-Schauss, D. Sabel, J. Niehren, and J. Schwinghammer. Observational Program Calculi and the Correctness of Translations. Rapport de recherche, Universität Frankfurt, Laboratoire d'Informatique Fondamentale de Lille - LIFL , LINKS - INRIA Lille - Nord Europe, May 2013.

40. R. H. Sloan and U. Buy. Reduction rules for time Petri nets. Acta Informatica, 33(5), Aug. 1996.

41. N. P. Smith and E. J. Crampin. Development of models of active ion transport for whole-cell modelling: cardiac sodium-potassium pump as a case study. Progress in biophysics and molecular biology, 85(2-3), 2004.

42. S. Soliman. Invariants and other structural properties of biochemical models as a constraint satisfaction problem. Algorithms for Molecular Biology, 7(1), May 2012.

43. S. Soliman, F. Fages, O. Radulescu, et al. A constraint solving approach to tropical equilibration and model reduction. In WCB-ninth Workshop on Constraint Based Methods for Bioinformatics, colocated with CP 2013, 2013.

44. I. Suzuki and T. Murata. A method for stepwise refinement and abstraction of Petri nets. Journal of Computer and System Sciences, 27(1), Aug. 1983.

45. R. Thomas. Regulatory networks seen as asynchronous automata: a logical description. Journal of theoretical biology, 153, 1991.

46. J. Uhlendorf, A. Miermont, T. Delaveau, G. Charvin, F. Fages, S. Bottani, G. Batt, and P. Hersen. Long-term model predictive control of gene expression at the population and single-cell levels. Proceedings of the National Academy of Sciences, 109(35), 2012.

47. R. Valette. Analysis of Petri nets by stepwise refinements. Journal of Computer and System Sciences, 1979. 


\section{A Soundness proof}

Theorem 1 (Soundness). For any $X \in\left\{\operatorname{Rev}, E n z, U s e, \mathrm{Cas}_{1}, \mathrm{Cas}_{2}\right\}$,

$$
\equiv_{\Omega, \mathcal{I}}^{X} \subseteq \equiv_{\Omega, \mathcal{I}}
$$

Proof. First, remember that a set $S$ is an attractor for $N$ iff $\forall s \in S . A c c_{N}(s)=S$, ie iff $S$ is closed under reduction and strongly connected. Let also recall that a network $N$ may diverge iff an attractor divergence is reachable.

Let $N \equiv \equiv_{\Omega, \mathcal{I}}^{X} N^{\prime}$, and $\mathcal{C}$ a context consistent with $\mathcal{I}$. We will show that $\mathcal{C}[N] \sim_{\Omega}$ $\mathcal{C}\left[N^{\prime}\right]$.

For the axiom Reversible (ie $N \equiv_{\Omega, I}^{R e v} N^{\prime}$ ).

First, we remark that for any reaction $r=s_{1} \rightarrow s_{2} \in \mathcal{C}[N]$, we can actually do $s_{1}[s / A] \underset{{\mathcal{C}\left[N^{\prime}\right]}^{*}}{*} s_{2}[s / A]$. Indeed, if $r \in R$, we can directly use $r[s / A] \in R[s / A]$. If $r=A \rightarrow s$ or $r=s \rightarrow A$, then $s_{1}[s / A]=s_{2}[s / A]$. And if $r \in \mathcal{C}$, then $A \notin \mathcal{I}$ implies $r=r[s / A]$. Then, by immediate recurrence, $s_{1} \underset{\mathcal{C}[N]}{\vec{N}^{*}} s_{2}$ implies $s_{1}[s / A] \underset{\mathcal{C}\left[N^{\prime}\right]}{*} s_{2}[s / A]$.

Reciprocally, for any reaction $r^{\prime}=s_{1}^{\prime} \rightarrow s_{2}^{\prime} \in \mathcal{C}\left[N^{\prime}\right]$, we can do $s_{1}^{\prime} \underset{\mathcal{C}[N]}{\rightarrow^{*}} s_{2}^{\prime}$. Indeed, if $r^{\prime} \in \mathcal{C}$, we can use it directly, and if $r^{\prime} \in R[s / A]$, then there is a $r=s_{1} \rightarrow s_{2} \in \mathcal{C}[N]$ s.t. $r^{\prime}=r[s / A]$, and we can do $\left.s_{1}^{\prime} \underset{\mathcal{C}[N]}{\vec{C}^{*}} s_{1} \underset{\mathcal{C}[N]}{\rightarrow} s_{2} \underset{\mathcal{C}[N]}{\vec{C}^{*}} s_{2}^{\prime}\right)$. Once again, by recurrence, $s_{1}^{\prime} \underset{\mathcal{C}\left[N^{\prime}\right]}{*} s_{2}^{\prime}$ implies $s_{1}^{\prime} \overrightarrow{\mathcal{C}[N]}^{*} s_{2}^{\prime}$, for any $s_{1}^{\prime}, s_{2}^{\prime}$.

Moreover, since $A \notin N^{\prime}$ and $A \notin \mathcal{C}$, we know by recurrence that for any $s_{1}$ reachable in $\mathcal{C}\left[N^{\prime}\right], A \notin s_{1}$.

Let $S$ be a reachable attractor for $\mathcal{C}[N]$, with a solution $s_{1} \in S$, and $S^{\prime}=$ $\operatorname{Acc}_{\mathcal{C}\left[N^{\prime}\right]}\left(s_{1}[s / A]\right)$. We will prove that $S^{\prime}$ is a reachable attractor for $\mathcal{C}[N]$. It is closed by definition, and $s_{1}$ reachable in $\mathcal{C}[p]$ implies $s_{1}[s / A]$ reachable in $\mathcal{C}\left[N^{\prime}\right]$. Take any $s_{2} \in S^{\prime}$, ie we can do $s_{1}[s / A] \overrightarrow{\mathcal{C}\left[N^{\prime}\right]}{ }^{*} s_{2}$. Then we can also so $s_{1} \overrightarrow{\mathcal{C}[N]}^{*} s_{1}[s / A] \underset{\mathcal{C}[N]}{\vec{C}^{*}} s_{2}$, so $s_{2} \in S$ (and $s_{1}[s / A] \in S$ too), and since $S$ is strongly connected, we can also do $s_{2} \overrightarrow{\mathcal{C}[N]}^{*} s_{1}[s / A]$. Since $A \notin s_{2}$, this implies $s_{2} \underset{\mathcal{C}\left[N^{\prime}\right]}{*} s_{1}[s / A]$. So $S^{\prime}$ is also strongly connected, so it is an attractor.

Reciprocally, let $S^{\prime}$ be a reachable attractor for $\mathcal{C}\left[N^{\prime}\right], s_{1} \in S^{\prime}$ a solution, and $S=A c c_{\mathcal{C}[N]}\left(s_{1}\right) . S$ is closed and reachable. Take $s_{2} \in S$, ie we can do $s_{1} \overrightarrow{\mathcal{C}[N]}^{*} s_{2}$. Then we can also so $s_{1}[s / A] \underset{\mathcal{C}\left[N^{\prime}\right]}{{ }^{*}} s_{2}[s / A]$. But $s_{1} \in S^{\prime}$ implies $A \notin s_{1}$, so $s_{1}[s / A]=s_{1} \in S^{\prime}$, and then $s_{2}[s / A] \in S^{\prime} . S^{\prime}$ is final, so we can do $s_{2}[s / A] \underset{\mathcal{C}\left[N^{\prime}\right]}{ }{ }^{*} s_{1}$. Then we can also do $s_{2} \underset{\mathcal{C}[N]}{\vec{C}^{*}} s_{2}[s / A] \underset{\mathcal{C}[N]}{\vec{C}^{*}} s_{1}$, so $S$ is strongly connected and is an attractor.

Moreover, $\Omega(A)=\Omega(s)$ implies $\Omega(S)=\Omega\left(S^{\prime}\right)$, so for any $O, N$ may converge 
to $O$ iff $N^{\prime}$ may converge to $O$.

If a solution $s_{1}$ is reachable and must diverge for $\mathcal{C}[N]$, then $s_{1} \underset{\mathcal{C}[p]}{*} s_{1}[s / A] \mathrm{im}$ plies that $s_{1}^{\prime}=s_{1}[s / A]$ must also diverge. And $s_{1}^{\prime}$ is reachable and must diverge for $\mathcal{C}\left[N^{\prime}\right]$ too.

Reciprocally, if a solution $s_{1}^{\prime}$ is reachable and must diverge for $\mathcal{C}\left[N^{\prime}\right]$, then it is directly reachable and must diverge for $\mathcal{C}[N]$ too.

So $\mathcal{C}[N] \sim_{\Omega} \mathcal{C}\left[N^{\prime}\right]$, and $N \equiv{ }_{\Omega, \mathcal{I}} N^{\prime}$.

Now consider the axiom ENZYME.

We show by recurrence that for any reachable $s$ in $\mathcal{C}[N]$ or $\mathcal{C}\left[N^{\prime}\right], s(A) \geq n$ : it is true initially by the conditions of the axiom. Assume it is true until a reachable solution $s^{\prime}$, and that we can do $s^{\prime} \underset{\mathcal{C}[N]}{\rightarrow} s$. Let $r$ be the reaction involved in this transition. If $r$ is a reaction of $\mathcal{C}$, then $A \notin \mathcal{I}$ implies $s(A)=s^{\prime}(A) \geq n$. If it is a reaction of $R, \operatorname{pr}_{r}(A) \geq 0$ implies $s(A) \geq s^{\prime}(A) \geq n$. And if $r=s_{1} \stackrel{n A}{\longrightarrow} s_{2}$, since $A \notin s_{1}$ we have $\operatorname{pr}_{r}(A) \geq 0$, and so $s(A) \geq s^{\prime}(A) \geq n$. The case for $\mathcal{C}\left[N^{\prime}\right]$ is identical.

So $s(A) \geq n$ for any reachable $s$. Then $s_{1} \stackrel{n A}{\longrightarrow} s_{2}$ is usable in a solution $s$ iff $s_{1} \rightarrow s_{2}$ is also usable in $s$. Since the two reactions make exactly the same change, then the networks have exactly the same reachability, and so they are congruent.

For the axiom USELESS.

Let $N \equiv{ }_{\Omega, \mathcal{I}}^{U s e} N^{\prime}$ and $\mathcal{C}$ be an admissible context with respect to $\mathcal{I}$. Let $N=$ $\left\langle s_{0}, R \cup\left\{s_{1} \stackrel{n A}{\longrightarrow} s_{2}\right\}\right\rangle$ and $N^{\prime}=\left\langle s_{0}, R \cup\left\{s_{1} \rightarrow s_{2}\right\}\right\rangle$.

Since $A$ cannot belong to the reactants of any reaction in $R$ and $\mathcal{C}$, for any solution $s$ any reaction $r$ applicable in $s+n A$ is also applicable in $s$. Hence, for any solution $s$ and $s^{\prime}$ :

$$
(s+n A) \underset{\mathcal{C}[N]}{\rightarrow^{*}}\left(s^{\prime}+n A\right) \text { iff } s \underset{\mathcal{C}\left[N^{\prime}\right]}{\rightarrow^{*}} s^{\prime}
$$

Moreover, the number of $A$ in the solutions of both networks cannot decrease. So in particular, any reachable solution in $\mathcal{C}[N]$ contains at least $n A$. Let $S$ be an attractor reachable from $\mathcal{C}[N]$, it must be of the form $S=\left\{\left(s^{\prime}+n A\right) \mid s^{\prime} \in S o l\right\}$. Then the set $S^{\prime}=\left\{s^{\prime} \mid\left(s^{\prime}+n A\right) \in S\right\}$ is an attractor reachable from $\mathcal{C}\left[N^{\prime}\right]$. Reversely, for any attractor $S^{\prime}$ reachable from $\mathcal{C}\left[N^{\prime}\right]$, the set $S=\left\{\left(s^{\prime}+n A\right) \mid s^{\prime} \in\right.$ $\left.S^{\prime}\right\}$ is an attractor reachable from $\mathcal{C}[N]$. The assumption of the axiom $\Omega(A)=\mathbf{0}$ implies $\Omega(S)=\Omega\left(S^{\prime}\right)$. Hence for any $O, \mathcal{C}[N]$ may converge to $O$ iff $\mathcal{C}\left[N^{\prime}\right]$ may converge to $O$.

Moreover, if a solution $s+n A$ must diverge and is reachable from $\mathcal{C}[N]$, then $s$ must diverge and is reachable from $\mathcal{C}\left[N^{\prime}\right]$, and reversely. So $\mathcal{C}[N]$ may diverge iff $\mathcal{C}\left[N^{\prime}\right]$ may diverge, and so $N$ and $N^{\prime}$ are attractor equivalent, that is $N \equiv_{\Omega, \mathcal{I}} N^{\prime}$.

For the axioms CASCADE 1 and $\mathrm{CASCADE}_{2}$.

For any $s_{1}, s_{2}$ with $A \notin s_{1}, A \notin s_{2}$ and s.t. $s_{1} \underset{\mathcal{C}[N]}{\rightarrow} s_{2}$, we can do $s_{1} \underset{\mathcal{C}\left[N^{\prime}\right]}{\rightarrow^{*}} s_{2}$. Indeed, we will first do the following operations on the transition $s_{1} \underset{\mathcal{C}[N]}{\rightarrow}{ }^{*} s_{2}$. 
Since $A \notin s_{1}, A \notin R$ and $A \notin \mathcal{I}$, we know that any occurrence of $\emptyset \stackrel{A}{\rightarrow} s^{\prime}$ $\left(\mathrm{CASCADE}_{1}\right)$ or $A \rightarrow s^{\prime}\left(\mathrm{CASCADE}_{2}\right)$ should be preceded by the reaction $\emptyset \stackrel{s}{\rightarrow} A$. Then we will put them directly after the $\emptyset \stackrel{s}{\rightarrow} A$. This is still a valid transition for $\mathcal{C}[p]$, since $A$ cannot be use in other useful reactions. We will also put the occurrences of $A \rightarrow \emptyset$ after the previous $A \rightarrow s^{\prime}$. So, for the axiom $\mathrm{CASCADE}_{1}$, the transition will be decomposable into the following parts : first a part without $A$, then creation of $A$ by $s$, some creations of $s^{\prime}$ with $A$ as an enzyme, and possibly degradation of $A$, then again a part without $A$, etc. For the axiom $\mathrm{CASCADE}_{2}$, the transition will alternate between parts without $A$, and parts with $\emptyset \stackrel{s}{\rightarrow} A$ followed by $A \rightarrow s^{\prime}$ or $A \rightarrow \emptyset$. Then, we can imitate the transition into $\mathcal{C}\left[p^{\prime}\right]$, by replacing the parts with $A$ by $\emptyset \stackrel{s}{\rightarrow} s^{\prime}$.

Rcpq, for any $s_{1}^{\prime}, s_{2}^{\prime}, s_{1}^{\prime} \underset{\mathcal{C}\left[N^{\prime}\right]}{\rightarrow^{*}} s_{2}^{\prime}$ implies $s_{1}^{\prime} \overrightarrow{\mathcal{C}[N]}^{*} s_{2}^{\prime}$ (we can replace $\emptyset \stackrel{s}{\rightarrow} s^{\prime}$ by $\emptyset \stackrel{s}{\rightarrow} A$, followed by $\emptyset \stackrel{A}{\rightarrow} s^{\prime}$ and $A \rightarrow \emptyset\left(\mathrm{CASCADE}_{1}\right)$, or by $\left.A \rightarrow s^{\prime}\left(\mathrm{CASCADE}_{2}\right)\right)$. Let $S$ be a reachable attractor for $\mathcal{C}[N]$ and $s_{1} \in S$. Then $s_{1}^{\prime}=s_{1} \backslash A$ is also in $S$, since $S$ is closed and we can apply $A \rightarrow \emptyset$. Let $S^{\prime}=A c c_{\mathcal{C}\left[N^{\prime}\right]}\left(s_{1}^{\prime}\right)$. It is closed and reachable. Let $s_{2}^{\prime} \in S^{\prime}$, ie $s_{1}^{\prime} \underset{\mathcal{C}\left[N^{\prime}\right]}{\vec{*}} s_{2}^{\prime}$. So we can also do $s_{1}^{\prime} \overrightarrow{\mathcal{C}[N]}^{*} s_{2}^{\prime}$, so $s_{2}^{\prime} \in S$, and we can do $s_{2}^{\prime} \overrightarrow{\mathcal{C}[N]}^{*} s_{1}^{\prime}$. We know that $A \notin s_{1}^{\prime}$, and $A \notin s_{2}^{\prime}$ (since $A \notin N^{\prime}$ and $A \notin \mathcal{I})$. So we can do $s_{2}^{\prime} \overrightarrow{\mathcal{C}\left[N^{\prime}\right]}{ }^{*} s_{1}^{\prime}$, so $S^{\prime}$ is strongly connected and an attractor. Rcpq, let $S^{\prime}$ be a reachable attractor for $\mathcal{C}\left[N^{\prime}\right]$, and $s_{1}^{\prime} \in S^{\prime}$. Then $s_{1}^{\prime}$ is also reachable in $\mathcal{C}[N]$. Let $S=A c c_{\mathcal{C}[N]}\left(s_{1}^{\prime}\right)$. It is closed and reachable. Let $s_{2} \in S$, ie $s_{1}^{\prime} \overrightarrow{\mathcal{C}[N]}^{*} s_{2}$. Then we can apply $A \rightarrow \emptyset$ and do $s_{2} \overrightarrow{\mathcal{C}[N]}^{*} s_{2}^{\prime}$ with $s_{2}^{\prime}=s_{2} \backslash A$. So $s_{1}^{\prime} \underset{\mathcal{C}[N]}{\vec{C}^{*}} s_{2}^{\prime}$ implies $s_{1}^{\prime} \underset{\mathcal{C}\left[N^{\prime}\right]}{\vec{x}^{*}} s_{2}^{\prime}$, so $s_{2}^{\prime} \in S^{\prime}$, and we can do $s_{2}^{\prime}{\overrightarrow{\mathcal{C}\left[N^{\prime}\right]}}^{*} s_{1}^{\prime}$ and $s_{2}^{\prime} \overrightarrow{\mathcal{C}}[N]^{*} s_{1}^{\prime}$. So we can also do $s_{2} \underset{\mathcal{C}[N]}{ }{ }^{*} s_{1}^{\prime}$, so $S$ is strongly connected and is an attractor.

For the observation, since $\Omega(A)=\emptyset$, we directly have $\Omega(S)=\Omega\left(S^{\prime}\right)$. So $\mathcal{C}[N]$ and $\mathcal{C}\left[N^{\prime}\right]$ may converge to the same set of observations.

If there is $s_{1}$ that must diverge in $N$, then $s_{1} \backslash A$ should also diverge in $N$, and it is reachable and must diverge in $N^{\prime}$ too. Rcpq, if $s_{1}^{\prime}$ must diverge in $N$, then it is directly reachable in $N$, and must diverge too. 